\title{
PREVALÊNCIA DE ADESÃO AO TRATAMENTO MEDICAMENTOSO POR DIABÉTICOS NO NORTE DO BRASIL
}

\author{
PREVALENCE OF MEDICATION ADHERENCE AMONG DIABETIC PATIENTS IN NORTHERN BRAZIL \\ PREVALENCIA DE CUMPLIMIENTO DE LA MEDICACIÓN EN DIABÉTICOS EN EL NORTE DE BRASIL \\ Tarcia Millene de Almeida Costa Barreto ${ }^{1}$ \\ Lanna Jennifer Silva Rodrigues ${ }^{2}$ \\ Jackeline da Costa Maciel ${ }^{3}$ \\ Dionisia Maria Sampaio 4 \\ Fabricio Barreto ${ }^{5}$
}

\section{RESUMO}

\begin{abstract}
$A$ diabetes mellitus (DM) apresenta altos índices de mortalidade; mesmo com os investimentos do Ministério da Saúde (MS), observa-se baixa adesão ao tratamento medicamentoso por diabéticos no Brasil, acarretando altos custos ao sistema de saúde. Assim, esta pesquisa teve por objetivo avaliar a prevalência de adesão ao tratamento medicamentoso por diabéticos e seus fatores correlacionados no Norte do Brasil. Recorreu-se ao método descritivo, analítico e quantitativo, baseado na aplicação de questionário semiestruturado e instrumentos de avaliação da adesão - os testes de Batalla e o de Morisky-Green. Dentre os entrevistados $(n=114)$ identificou-se: maior prevalência de DM em idosos $( \pm 62,17$ anos $)$, mulheres $(78,00 \%)$, indivíduos casados (40,35\%), indivíduos com baixa escolaridade e renda familiar < 1 salário-mínimo (63,16\%). 0 tempo de diagnóstico foi de 8,28 $\pm 7,75$ anos. 0 teste de Batalla indicou 29,82\% de adesão e o teste de Morisky-Green indicou 27,19\% de adesão. Quanto aos fatores que podem interferir na adesão ao tratamento, observou-se que $87,10 \%$ têm cumprido o intervalo entre as consultas médicas, 58,06\% apresentam comorbidades e 90,32\% não participam de grupos de apoio. Esta pesquisa busca contribuir com o desenvolvimento de estratégias de promoção à saúde por meio de políticas públicas.
\end{abstract}

Palavras-chave: Diabetes Mellitus; Adesão ao Tratamento; Autocuidado; Educação em Saúde.

\footnotetext{
1. Enfermeira. Mestre em Ciências da Saúde pela Universidade Federal de Roraima (UFRR). Professora na UfRR. Boa Vista (RR), Brasil.

2. Enfermeira graduada pela UFRR. Boa Vista (RR), Brasil.

3. Biomédica. Doutora em Ciências Biológicas pela Universidade Federal de Pernambuco (UFPE). Professora na UFRR. Boa Vista (RR), Brasil.

4. Aluna de graduação em Enfermagem na UFRR. Boa Vista (RR), Brasil.

5. Enfermeiro. Mestre em Ciências da Saúde pela UfRR. Professor na UFRR. Boa Vista (RR), Brasil.
} 


\section{ABSTRACT}

Diabetes mellitus (DM) has high mortality rates; even with investments led by the Ministry of Health (MoH), there is low medication adherence among diabetic patients in Brazil, causing high costs to the health system. Thus, this research aimed to evaluate the prevalence of medication adherence among diabetic patients and its correlated factors in Northern Brazil. The descriptive, analytical, and quantitative method was used, based on the application of a semi-structured questionnaire and instruments for assessing medication adherence - the Batalla and Morisky-Green tests. Among respondents $(n=114)$, we identified: a higher prevalence of DM in the elderly ( \pm 62.17 years), women $(78.00 \%)$, married individuals $(40.35 \%)$, individuals with low school education and family income $<1$ minimum wage (63.16\%). The time since diagnosis was $8.28 \pm 7.75$ years. The Batalla test pointed out $29.82 \%$ of adherence and the Morisky-Green test pointed out $27.19 \%$ of adherence. Regarding the factors that may interfere with medication adherence, $87.10 \%$ have met the interval between medical appointments, 58.06\% have comorbidities, and $90.32 \%$ do not participate in support groups. This research seeks to contribute to developing health promotion strategies through public policies.

Keywords: Diabetes Mellitus; Medication Adherence; Self Care; Health Education.

\section{RESUMEN}

La diabetes mellitus (DM) tiene altas tasas de mortalidad; incluso con inversiones del Ministerio de Salud (MS), existe un bajo cumplimiento de la medicación entre los diabéticos en Brasil, lo que ocasiona altos costos para el sistema de salud. Por lo tanto, esta investigación tuvo como objetivo evaluar la prevalencia de cumplimiento de la medicación entre diabéticos y sus factores correlacionados en el Norte de Brasil. Se utilizó el método descriptivo, analítico y cuantitativo, basado en la aplicación de un cuestionario semi-estructurado e instrumentos para evaluar el cumplimiento de la medicación - las pruebas de Batalla y MoriskyGreen. Entre los encuestados $(n=114)$, identificamos: una mayor prevalencia de DM en ancianos $( \pm 62,17$ años), mujeres $(78,00 \%)$, personas casadas $(40,35 \%)$, personas con educación escolar baja e ingreso familiar < 1 salario mínimo (63,16\%). El tiempo desde el diagnóstico fue de 8,28 \pm 7,75 años. La prueba de Batalla señaló 29,82\% de cumplimiento y la prueba de MoriskyGreen señaló $27,19 \%$ de cumplimiento. Con respecto a los factores que pueden interferir con el cumplimiento de la medicación, $87,10 \%$ han cumplido el intervalo entre citas médicas, 58,06\% tienen comorbilidades y $90,32 \%$ no participan en grupos de apoyo. Esta investigación busca contribuir al desarrollo de estrategias de promoción de la salud a través de políticas públicas.

Palabras clave: Diabetes Mellitus; Cumplimiento de la Medicación; Autocuidado; Educación en Salud.

\section{INTRODUÇÃO}

De acordo com os dados do Ministério da Saúde (MS), vem ocorrendo um aumento significativo do número de óbitos por doenças crônicas não transmissiveis no Brasil, entre elas a diabetes mellitus (DM). Em termos estatísticos mundiais, o Brasil ocupa a $8^{a}$ posição no ranking de prevalência de DM, com prevalência de $4,60 \%$, e estima-se que até 2030 ocupe a $6^{\text {a }}$ posição, chegando a atingir $11,30 \%$ da populaçã $0^{1}$.

No Brasil, o MS estima que $12,00 \%$ da população sofra de DM. Em Roraima, esse índice é de $10,60 \%$ da população - na capital desse estado, Boa Vista, a prevalência cai para $8,42 \%{ }^{1}$.

A estimativa é que nos próximos anos esses números cresçam de modo significativo, considerando que a população vem envelhecendo e há persistência de hábitos de vida não saudáveis, como: consumo abusivo de álcool; sedentarismo decorrente do desenvolvimento tecnológico; tabagismo; e alimentação inadequada ${ }^{2,3}$.
A DM é uma condição de saúde considerada sensível à atenção primária à saúde (APS), ou seja, evidências demonstram que o bom manejo desse problema ainda na APS evita hospitalizações e mortes decorrentes de complicações cardiovasculares e cerebrovasculares. Cerca de 60,00\% a $80,00 \%$ dos casos demandam apenas medidas de promoção e prevençã $0^{3-6}$.

No entanto, a adesão ao tratamento depende de fatores que vão além da criação de políticas de saúde, como: fatores sociais; fatores econômicos; comprometimento da equipe de saúde; e conhecimento do paciente acerca de sua doença. É com base na eficácia dessa adesão que se obtém respostas nos casos - seja para manutenção da saúde ou para complicações secundárias ${ }^{3,5,7,8}$.

Este estudo avalia a prevalência de adesão ao tratamento medicamento por diabéticos e correlaciona os fatores que interferem na manutenção do autocuidado e suas implicações, assim como o perfil socioeconômico e as características epidemiológicas. 


\section{METODOLOGIA}

Trata-se de estudo de corte transversal, com análise descritiva e quantitativa, desenhada para avaliar a prevalência de adesão ao tratamento por portadores de DM na APS de Boa Vista.

0s sujeitos da pesquisa foram os 4.711 habitantes de Boa Vista cadastrados no Sistema Único de Saúde (SUS) como portadores de DM - assistidos em unidades básicas de saúde (UBS).

Para o cálculo do tamanho amostral, a prevalência de adesão ao tratamento por diabéticos foi estimada em $6,70 \%$, com base nos resultados de estudo realizado com pacientes ambulatoriais ${ }^{6}$. Considerando intervalo de confiança de $95,00 \%$ e erro amostral de 5,00\%, obteve-se o tamanho amostral mínimo de 89 indivíduos. Supondo uma perda de até $5,00 \%$ de dados, o tamanho da amostra final foi de 94 participantes.

Foi utilizado o método de amostragem aleatório por conglomerado, assumindo cada UBS de Boa Vista como um conglomerado amostral. As UBS foram enumeradas e selecionadas randomicamente, pela geração de sequência de números aleatórios.

A coleta de dados primários foi realizada com todos os usuários que atenderem aos critérios de inclusão, em entrevista face a face, com duração média de 30 minutos. Para a avaliação da adesão ao tratamento pelos usuários, foram aplicados testes padronizados, amplamente adotados em pesquisas que avaliam adesão de pacientes com hipertensão e DM: Batalla e Morisky-Green ${ }^{4,6} .0$ teste de Batalla é composto por 3 perguntas e avalia a adesão ao tratamento por meio do conhecimento do usuário acerca de sua doença. Já o teste de Morisky-Green é composto por 4 perguntas e mensura a adesão por meio das atitudes dos pacientes durante a utilização dos medicamentos. Ambos os testes exigem que 0 usuário responda corretamente a todas as perguntas para que seja considerado aderente ao tratamento.

Quanto aos aspectos éticos, esta pesquisa foi aprovada pelo Comitê de Ética em Pesquisa da Universidade Federal de Roraima, sob o Parecer n. 1.350.595/2015, e obteve autorização da Secretaria Municipal de Saúde de Boa Vista para utilização de seu banco de dados e realização das visitas às UBS.

\section{RESULTADOS}

Foram abordados 114 diabéticos atendidos nas UBS de Boa Vista, o que corresponde a 2,41\% dos portadores de DM do município. 0 número de usuários ultrapassou o estimado, pois a abordagem foi realizada por conglomerados - cada UBS foi definida como um conglomerado.

Verificou-se que, dentre os pacientes entrevistados, a maioria consistia em: idosos (média de idade de 62,17 $\pm 11,83$ anos); mulheres $(78,00 \%)$; indivíduos casados $(40,35 \%)$; indivíduos com baixa escolaridade (Ensino Fundamental incompleto - 57,89\%); indivíduos com renda familiar < 1 salário-mínimo $(63,16 \%)$, sendo $50,00 \%$ deles aposentados.

0 tempo de diagnóstico relatado da doença foi de $8,28 \pm 7,75$ anos. Quanto à periodicidade das consultas médicas, observou-se que $37,72 \%$ realizam a cada 3 meses e $32,46 \%$ realizam esporadicamente. Em relação à aferição da glicemia, apenas $16,67 \%$ verificam diariamente e $51,75 \%$ esporadicamente. Dentre os entrevistados, 56,65\% relataram ter, além de DM, hipertensão arterial. Quanto aos fatores de predisposição, $50,00 \%$ são sedentários, $21,93 \%$ são consumidores de álcool e 10,53\% são tabagistas. Quanto à participação em grupos de apoio, 11,40\% afirmaram participar, porém, não na UBS, mas em programas de atenção à terceira idade (Tabela 1 ).

Tabela 1 - Características de diabéticos atendidos em UBS. Boa Vista, 2015-2016.

\begin{tabular}{|c|c|c|}
\hline Variável & Media \pm DP(anos) & $\%(n)$ \\
\hline Tempo de diagnóstico & $8,28 \pm 7,75$ & \\
\hline \multicolumn{3}{|c|}{ Periodicidade da consulta } \\
\hline Mensal & & $22,80(26)$ \\
\hline Bimestral & & $5,26(6)$ \\
\hline Trimestral & & $37,72(43)$ \\
\hline Semestral & & $1,75(2)$ \\
\hline Esporádico & & $32,46(37)$ \\
\hline \multicolumn{3}{|c|}{ Periodicidade com que verifica a glicemia } \\
\hline Diária & & $16,67(19)$ \\
\hline Quinzenal & & $8,77(10)$ \\
\hline Mensal & & $12,28(14)$ \\
\hline Trimestral & & $7,89(9)$ \\
\hline Anual & & $0,88(1)$ \\
\hline Esporádico & & $51,75(59)$ \\
\hline Nunca & & $1,75(2)$ \\
\hline
\end{tabular}




\begin{tabular}{|c|c|c|}
\hline Variável & Media \pm DP(anos) & $\%(n)$ \\
\hline $\begin{array}{l}\text { Medicamentos utilizados } \\
\text { Glavus } \\
\text { Glibenclamida } \\
\text { Glicazida } \\
\text { Insulina NPH } \\
\text { Insulina regular } \\
\text { Meritor } \\
\text { Metformina }\end{array}$ & & $\begin{aligned} 0,88 & (1) \\
32,56 & (37) \\
1,76 & (2) \\
1,76 & (2) \\
4,38 & (5) \\
0,88 & (1) \\
28,94 & (33)\end{aligned}$ \\
\hline $\begin{array}{l}\text { Medicamentos utilizados } \\
\text { Tarjeta duo } \\
\text { Desconhece }\end{array}$ & & $\begin{array}{cc}0,88 & (1) \\
28,94 & (33)\end{array}$ \\
\hline $\begin{array}{l}\text { Outras morbidades } \\
\text { Artrite } \\
\text { Artrose } \\
\text { Bronquite } \\
\text { Asma } \\
\text { Colesterol alto } \\
\text { Dislipidemia } \\
\text { Hérnia } \\
\text { Hipertensão } \\
\text { Labirintite } \\
\text { Osteoporose } \\
\text { Parkinson } \\
\text { Câncer } \\
\text { Nenhuma }\end{array}$ & & $\begin{array}{c}0,88(1) \\
2,63(3) \\
0,88(1) \\
0,88(1) \\
3,50(4) \\
0,88(1) \\
0,88(1) \\
56,65(68) \\
1,76(2) \\
4,38(5) \\
1,76(2) \\
0,88(1) \\
32,46(37)\end{array}$ \\
\hline $\begin{array}{l}\text { Fatores de predisposição a } \\
\text { Tabagista } \\
\text { Etilista } \\
\text { Sedentário }\end{array}$ & & $\begin{array}{c}10,53(12) \\
21,93(25) \\
50(57)\end{array}$ \\
\hline Participa de grupo de apoio & & $11,40(13)$ \\
\hline
\end{tabular}

Nota: DP = desvio padrão.

Fonte: Elaborada pelos autores.

Quanto à frequência de adesão ao tratamento medicamentoso, observa-se na Tabela 2 que, como estabelece o teste de Batalla, somente $29,82 \%$ dos diabéticos são aderentes. Em relação às perguntas, $41,23 \%$ indicou corretamente os órgãos que podem ser afetados pela doença - sendo mais citados os olhos e os rins.

Tabela 2 - Adesão ao tratamento medicamentoso de diabéticos atendidos em UBS, segundo o teste de Batalla. Boa Vista, 2015 2016.

\begin{tabular}{lc}
\hline Perguntas & $\begin{array}{c}\text { Respostas corretas } \\
\%(\mathbf{n})\end{array}$ \\
\hline Afirmaram que sua doença é para a vida toda & $79,82(91)$ \\
Afirmaram que pode ser controlada com dieta ou medicamentos & $95,61(109)$ \\
Citaram dois órgãos que podem ser afetados pela doença & $41,23(47)$ \\
Grau de adesão & $29,82(37)$ \\
\hline
\end{tabular}

Fonte: Elaborada pelos autores.

Frequência semelhante foi obtida com o teste de Morisky-Green, com adesão de $27,19 \%$ - as principais causas para não adesão foram perda do horário e não tomar quando o medicamente quando causa mal-estar. A primeira causa caracteriza não intencionalidade, porém, a segunda causa demonstra comportamento intencional diante da farmacoterapia (Tabela 3).

Tabela 3 - Adesão ao tratamento medicamentoso de diabéticos atendidos em UBS, segundo o teste de Morisky-Green. Boa Vista, 2015-2016.

\begin{tabular}{lc} 
Perguntas & $\begin{array}{c}\text { Respostas corretas } \\
\%(\mathbf{n})\end{array}$ \\
\hline Não têm dificuldade para tomar o medicamento & $78,07(89)$ \\
Tomam na hora indicada & $53,51(61)$ \\
\hline
\end{tabular}




\begin{tabular}{|c|c|}
\hline Perguntas & $\begin{array}{c}\text { Respostas corretas } \\
\% \text { (n) }\end{array}$ \\
\hline Não deixam de tomar o medicamento quando se encontram bem & $77,19(88)$ \\
\hline Deixam de tomar a medicação se alguma vez se sentirem mal & $74,56(85)$ \\
\hline Grau de adesão & $27,19(31)$ \\
\hline
\end{tabular}

Fonte: Elaborada pelos autores.

A investigação do conhecimento acerca do tratamento da doença (Tabela 4) mostrou que 59,00\% dos entrevistados sabem o nome de seu medicamento e $61,95 \%$ conhecem a finalidade de seu uso. Quando questionados quanto à orientação sobre o tratamento, 67,54\% afirmaram que sim, sendo o médico o principal transmissor dessas informações. Ao ser questionados sobre as dificuldades para manter o tratamento, foi relatada como principal causa a manutenção da dieta $(53,51 \%)$, seguida da realização de atividade física $(24,56 \%)$.

Tabela 4 - Conhecimento de diabéticos atendidos em UBS sobre o tratamento de sua doença. Boa Vista, $2015-2016$.

\begin{tabular}{lc}
\hline Perguntas & $\%(\mathbf{n})$ \\
\hline Afirmaram saber o nome de todos os medicamentos & $59(47)$ \\
Afirmaram saber a dose de todos os medicamentos & $60,87(59)$ \\
Afirmaram saber o intervalo entre as doses dos medicamentos & $88,04(81)$ \\
Afirmaram saber para que servem os medicamentos & $61,95(57)$ \\
Afirmaram ter recebido orientação sobre o tratamento & $67,54(77)$ \\
Profissional que orientou & $5,19(4)$ \\
Enfermeiro & $92,20(71)$ \\
Médico & $2,60(2)$ \\
Nutricionista & \\
Dificuldades relatadas quanto ao tratamento & $16,67(19)$ \\
Questões econômicas & $13,15(15)$ \\
Falta de informação & $53,51(61)$ \\
Dieta alimentar & $0,88(1)$ \\
Deslocamento & $24,56(28)$ \\
Atividade física & $2,63(3)$ \\
Medicamentos & $0,88(1)$ \\
Falta de tempo &
\end{tabular}

Fonte: Elaborada pelos autores.

Quando questionados sobre os profissionais que os acompanham na UBS, o médico foi apontado em $42,98 \%$ das respostas, seguido pelo agente comunitário de saúde (ACS), com 31,57\%. Um fato que chamou atenção foi a realização de atividades de educação em saúde, onde $82,46 \%$ dos entrevistados afirmaram que sua unidade não realiza nenhuma atividade de educação em saúde, 15,79\% afirmaram que a unidade realiza palestras periódicas e somente $1,76 \%$ relataram que a unidade dispõe de grupo de apoio. Sobre a disponibilidade do medicamento, apenas $25,44 \%$ dos entrevistados apontaram dificuldades para receber 0 medicamento na UBS.

Quanto aos fatores que podem interferir na adesão ao tratamento, foi avaliada a associação entre adesão (teste de Morisky-Green) e componentes do controle metabólico da DM. Observou-se que, dentre os aderentes ao tratamento,
$87,10 \%$ têm cumprido o período de 3 meses para consultas médicas; no entanto, 58,06\% têm comorbidades, $90,32 \%$ não participam de grupos de apoio e $51,61 \%$ relataram não praticar atividade física. Entre os não aderentes ao tratamento, verificou-se que $74,69 \%$ estão em dia com suas consultas médicas, $71,08 \%$ têm comorbidades, $87,95 \%$ não participam de grupos de apoio e 50,60\% não praticam atividades físicas. A associação que se mostrou significativa foi o maior percentual de comorbidades entre os pacientes não aderentes ao tratamento $(71,08 \%)$ em comparação aos aderentes $(58,06 \%)$, evidenciando que a adesão ao tratamento traz benefícios aos pacientes.

Quanto à avaliação da relação entre adesão ao tratamento (teste de Batalla) e o nível de escolaridade dos entrevistados, observou-se que com níveis baixos de instrução a adesão se manteve baixa, porém, o dado se replica nos usuários com maior nível de escolaridade, por exemplo, dentre os que se 
declararam analfabetos, $76,47 \%$ não aderem ao tratamento; dentre os que têm Ensino Fundamental incompleto, 92,16\% também não aderem ao tratamento; somente dentre os que têm Ensino Fundamental completo se observou prevalência entre pacientes aderentes ao tratamento $(89,96 \%)$. Já dentre os que têm Ensino Superior se mantém o padrão de não adesão, com proporção de $66,67 \%$, apesar de haver pequeno número de entrevistados com esse perfil.

Ao avaliar a associação entre adesão ao tratamento (segundo Morisky-Green) e o sexo dos entrevistados, observou-se que o sexo dos usuários não influenciou significativamente nos resultados: $41,94 \%$ para homens e $58,06 \%$ para mulheres, com valor de $p=0,96$. Isso também ocorre no teste de Batalla, com proporção de $40,54 \%$ para homens e $59,46 \%$ para mulheres.

Também foi analisada a relação entre a adesão ao tratamento e o tempo de tratamento, sendo separados em 2 grupos: < 10 anos de tratamento e > 10 anos - valores proporcionais de $24,13 \%$ e $37,04 \%$ foram observados no teste de Batalla e $28,73 \%$ e $44,44 \%$ no teste de Morisky-Green.

\section{DISCUSSÕES}

Este estudo foi proposto para identificar a prevalência e correlacionar os fatores que interferem na adesão ao tratamento de diabéticos atendidos em APS de Boa Vista. Por ser uma pesquisa com foco exclusivo no tema, a garantia de um $n$ amostral representativo da população e a utilização de testes validados para adesão a tratamento da DM constituem fatores positivos.

Observamos prevalência de mulheres $(68,42 \%)$, corroborando o perfil nacional dos portadores de $\mathrm{DM}^{4,6}$. ressalta-se que as mulheres apresentam maior capacidade de adaptação à condição de saúde, apesar de não perceberem o apoio social no tratamento. Tal fato pode estar relacionado às inúmeras cobranças sociais que recaem sobre a mulher - como cuidar dos filhos e do lar, além de muitas vezes trabalhar fora de casa - e à ausência de sintomas da DM, o que faz com que os participantes sociais não percebam a doença como real e mantenham as cobranças sobre os portadores, sejam homens ou mulheres ${ }^{9,10}$.

Quanto à escolaridade, 57,89\% dos entrevistados tinham apenas 0 Ensino Fundamental incompleto. Tal fator pode desencadear menor compreensão da doença e, assim, menor adesão ao tratamento. Entretanto, com os resultados deste estudo, o mais prudente seria afirmar que a escolaridade interfere somente no local de busca pelo tratamento, ou seja, quanto menor o grau de escolaridade, menor o poder aquisitivo e maior a busca por atendimento nos serviços públicos. Tal afirmação pode ser ratificada pelos resultados da variável renda familiar, haja vista que $63,16 \%$ dos

\section{...a escolaridade interfere somente no local de busca pelo tratamento...}

entrevistados têm renda inferior a 1 salário-mínimo 4,9,11,12.

Quanto aos medicamentos utilizados pelos pacientes, observou-se que os hipoglicemiantes orais de maior prevalência foram: glibenclamida $(32,56 \%)$, metformina $(28,94 \%)$ e insulina regular $(4,28 \%)$. Salienta-se que a utilização de hipoglicemiantes orais favorece a baixa adesão, tendo em vista a imensa possibilidade de esquecimento $0^{6,11,13-15}$.

De acordo com o MS, a APS deve seguir critérios para acompanhamento dos pacientes com doenças crônicas, que devem ir além da realização de consultas médicas e prescrições. Deve existir interesse em orientar o paciente para mudança de estilo de vida, manutenção dos padrões glicêmicos dentro da normalidade e prevenção de complicações, sempre buscando um cuidado integral e longitudinal, com garantia de qualidade de vida ao diabético $0^{2,15,16}$.

0 portador de DM precisa receber orientação quanto ao tratamento medicamentoso, porém, deve ser mantido acompanhamento sistemático pela equipe multiprofissional de saúde, com consultas rotineiras, a depender da estratificação de risco de complicações de cada paciente ${ }^{16,17}$.

Neste estudo, observou-se que grande parte dos usuários tem mantido padrão apropriado para realização de consultas, fato evidenciado pelas proporções de consultas mensais $(22,80 \%)$, bimestrais $(5,26 \%)$ e trimestrais $(37,72)$. No entanto, quando eles foram questionados sobre a participação em grupos de apoio, apenas $11,40 \%$ responderam positivamente, porém, deve-se registrar que somente 2 informaram que esses grupos ocorrem na UBS, os demais participam de programas sociais voltados a idosos. Dessa forma, pode-se concluir que as consultas são realizadas apenas para renovação de prescrição medicamentosa, um achado que corrobora tal afirmação é que $42,98 \%$ dos entrevistados apontaram o médico como responsável pelo acompanhamento de seu tratamento na UBS e apenas $11,40 \%$ apontaram o enfermeiro. Entende-se que o atendimento vem ocorrendo, mas não em sua totalidade, como preconizado nas orientações do $\mathrm{MS}^{18}$.

Como estratégia, para quantificar a adesão ao tratamento medicamentoso foram adotados dois testes de adesão: o teste de Morisky-Green e o teste de Batalla - utilizados em diversas pesquisas, demonstrando bons resultados. 0 teste de Morisky-Green pode indicar quais pacientes devem receber 
reforços educativos, assistência farmacêutica e atendimento multidisciplinar ${ }^{19}$.

Ao aplicar o teste de Morisky-Green, obteve-se uma proporção de adesão de $27,19 \%$ dos usuários, prevalência bem abaixo do padrão recomendado por outros autores, que seria em torno de $80,00 \%{ }^{19}$. Valores menores foram encontrados, com adesão de $15,00 \%^{6}$ e $6,74 \%^{20}$. Dentre as perguntas do teste, $53,51 \%$ dos entrevistados respondeu que tomam o medicamento na hora indicada e $74,56 \%$ deixam de tomar 0 medicamento quando causa mal-estar. Resultados similares também foram observados em outros estudos 4,6,14,19,20.

Ações de promoção à saúde, como encontros mensais, palestras e visitas domiciliares, seriam relevantes para mudança desse cenário, uma vez que a compreensão do tratamento facilita sua execução, especialmente em pacientes com doenças crônicas e "silenciosas" como a DM.

Embora os testes de Morisky-Green e de Batalla apresentem características divergentes, a aplicação dos dois juntos auxilia na obtenção de uma resposta mais fidedigna. Dentre os fatores que poderiam estar relacionados a essa baixa adesão estariam o sexo, a baixa escolaridade e o controle metabólico da DM. Quanto ao sexo, os homens apresentaram maior adesão em ambos os testes, no entanto, vale ressaltar que, levando em consideração o teste de hipótese, fica evidente que não há relação entre sexo e adesão, corroborando pesquisas nacionais - onde a adesão não apresenta diferenças significativas entre os sexos ${ }^{4,21}$.

No teste das variáveis adesão e tempo de tratamento, não foi observada diferença significativa entre os dados, considerando o valor de $p$ encontrado. A perspectiva é que, com o passar dos anos, os pacientes diminuam a adesão ao tratamento, o que contribui com o aumento das complicações ${ }^{19,21,22}$. Pode-se dizer que, por se tratar de uma doença silenciosa, ao longo dos anos, o indivíduo reconhece os sintomas de complicações e agravo da doença e não mais executa o tratamento com eficiência.

Inúmeros são os fatores que podem interferir na adesão ao tratamento, dentre eles os culturais, a origem da população estudada, a relação com a equipe de saúde e com a família e que em cada 10 portadores de doenças crônicas, cerca de 3 apresentam alguma característica de descontinuidade da terapia medicamentosa, podendo decorrer de orientação inadequada $13,22,23$.

Foram avaliados o conhecimento dos pacientes sobre seu tratamento e suas principais dificuldades. Observou-se que $67,54 \%$ dos pacientes afirmaram ter recebido orientação sobre seu tratamento e, destes, $77,17 \%$ foram atendidos por médicos e, no entanto, $41,00 \%$ não souberam informar 0 nome do medicamento que tomam e $38,08 \%$ não souberam para que serve o medicamento. Dessa forma, percebe-se que há deficiência quanto à orientação aos pacientes. Essas lacunas no conhecimento do tratamento afetam diretamente a adesão - elas poderiam ser suprimidas por uma abordagem compartilhada da doença entre profissional da saúde e paciente $23-25$.

\section{CONSIDERAÇÕES FINAIS}

Nesta pesquisa, evidenciou-se a baixa adesão ao tratamento medicamentoso por parte dos diabéticos em Boa Vista, em relação à proporção recomendada pelo MS e identificou-se uma relação entre a não adesão e a não realização de atividades de autocuidado, como a prática de atividades físicas, mudança no estilo de vida, inferindo padrão alimentar, consumo de bebidas alcoólicas, tabagismo e outros. Destacam-se três pontos como influenciadores na adesão dos pacientes ao tratamento: falta de conhecimento da doença; falta de compreensão do tratamento; e não participação em atividades de educação em saúde.

As ações de educação em saúde, por parte da UBS afetam diretamente a compreensão da doença e a aceitação do tratamento, desde que sejam realizadas de modo adequado, observando os múltiplos fatores que interferem na adesão ao tratamento medicamentoso da DM. 0 processo de autocuidado deve ser estimulado mediante orientação, cuidado sistemático, visitas periódicas, averiguação de medicamentos e controle dos índices glicêmicos.

Considerando que as doenças crônicas apresentam características de baixa adesão ao tratamento e, em especial, a DM, que tem manifestação silenciosa, mostra-se necessário o desenvolvimento de estratégias que busquem o envolvimento da comunidade e da família, com o intuito de facilitar a compreensão do processo de adoecimento e promoção do bem-estar. Não há como o paciente compreender seu tratamento medicamentoso se não compreender as razões de aderir a ele, os efeitos positivos e negativos de cada medicamento.

Por fim, ressalta-se que este estudo constitui iniciativa pioneira em Boa Vista e aponta possibilidades para reestruturar o planejamento de ações estratégicas nas UBS, tendo em vista os indicadores que avaliou. Além disso, nossas conclusões podem prover subsídios para investigações junto a comunidades de municípios com porte e perfil sanitário semelhante.

$$
\begin{gathered}
\text {...lacunas no } \\
\text { conhecimento do } \\
\text { tratamento afetam } \\
\text { diretamente a } \\
\text { adesão... }
\end{gathered}
$$




\section{CONTRIBUIÇÃO DOS AUTORES}

Tarcia Millene de Almeida Costa Barreto contribuiu com o delineamento e a realização da pesquisa e a redação do manuscrito. Lanna Jennifer Silva Rodrigues contribuiu com a coleta de dados em campo. Jackeline da Costa Maciel contribuiu com o delineamento da pesquisa e a revisão crítica do manuscrito. Dionisia Maria Sampaio contribuiu com a coleta de dados em campo. Fabrício Barreto contribuiu com a revisão estatística.

\section{REFERÊNCIAS}

1. Organização Mundial da Saúde. Cuidados inovadores para condições crônicas: componentes estruturais de ação. Brasília (DF): 0MS; 2003.

2. Brasil. Saúde Brasil 2010: uma análise da situação de saúde e da agenda nacional e internacional de prioridades em saúde. Brasília (DF): Ministério da Saúde; 2011.

3. Alfradique ME, Bonolo PF, Dourado I, Costa MFL, Macinko $\mathrm{J}$, Mendonça CS, et al. Internações por condições sensíveis à atenção primária: a construção da lista brasileira como ferramenta para medir o desempenho do sistema de saúde (Projeto ICSAP - Brasil). Cad Saúde Pública [serial on the internet]. 2009 [cited 2017 Dec 11];25(6):1337-49. Available from: http://www.scielo.br/pdf/csp/v25n6/16.pdf

4. Carvalho ALM, Leopoldino RWD, Silva JEG, Cunha CP. Adesão ao tratamento medicamentoso em usuários cadastrados no Programa Hiperdia no município de Teresina (PI). Ciênc Saúde Colet [serial on the internet]. 2012 [cited 2017 Dec 11];17(7):1885-92. Available from: http://www. scielo.br/pdf/csc/v17n7/28.pdf

5. Iser BPM. Prevalência de diabetes autorreferido no Brasil: resultados da Pesquisa Nacional de Saúde 2013. Epidemiol Serv Saúde [serial on the internet]. 2015 [cited 2017 Dec 11];24(2):305-14. Available from: http://www.scielo.br/ $\mathrm{pdf} / \mathrm{ress} / \mathrm{v} 24 \mathrm{n} 2 / 2237-9622-$ ress-24-02-00305.pdf

6. Santos FS, Oliveira KR, Colet CF. Adesão ao tratamento medicamentoso pelos portadores de diabetes mellitus atendidos em uma unidade básica de saúde no município de Ijuí/RS: um estudo exploratório. Rev Ciênc Farm Básica Apl [serial on the internet]. 2010 [cited 2017 Dec 11];31(3):2237. Available from: http://serv-bib.fcfar.unesp.br/seer/index. php/Cien_Farm/article/view/1572/992

7. Brasil. Estratégias para o cuidado da pessoa com doença crônica: diabetes mellitus. Brasília (DF): Ministério da Saúde; 2013.

8. Klafke A, Duncan BB, Rosa RS, Moura L, Malta DC, Schmidt MI. Mortalidade por complicações agudas do diabetes melito no Brasil, 2006-2010. Epidemiol Serv Saúde [serial on the internet]. 2014 [cited 2017 Dec 11];23(3):455-62. Available from: $\quad$ http://www.scielo.br/pdf/ress/v23n3/1679-4974ress-23-03-00455.pdf
9. Santos KP, Costa MCM, Rios MC, Rios PSS. Adesão a farmacoterapia em pacientes cadastrados no Hiperdia em uma unidade básica de saúde em Aracaju, SE. Infarma: Ciências Farmacêuticas [serial on the internet]. 2014 [cited 2017 Dec 11];26(4):233-9. Available from: http://revistas.cff.org.br/ ?journal=infarma\&page $=$ article \&op $=$ view \& path $\% 5 \mathrm{~B} \% 5 \mathrm{D}=672$ \&path $\% 5 B \% 5 D=p d f \_19$

10. Cardoso AF, Queiros P, Ribeiro CF. Intervenções para a aquisicão do autocuidado terapêutico da pessoa com diabetes mellitus: revisão sistemática da literatura. Rev Port Saúde Pública [serial on the internet]. 2015 [cited 2017 Dec 11];33(2):246-55. Available from: http://www.sciencedirect. com/science/article/pii/S0870902515000322

11. Estancial CS, Marini DC, Aderência de diabéticos ao tratamento medicamentoso. Foco: Caderno de Estudos e Pesquisas [serial on the internet]. 2013 [cited 2017 Dec 11];4(5):27-40. Available from: http://www.revistafoco.inf. br/index.php/FocoFimi/article/view/31/34

12. Groff DPG, Simões PWTA, Fagundes ALSC. Adesão ao tratamento dos pacientes diabéticos tipo II usuários da Estratégia Saúde da Família situada no bairro Metropol de Criciúma, SC. ACM Arq Catarin Med [serial on the internet]. 2011 [cited 2017 Dec 11];40(3):43-8. Available from: http:// www.acm.org.br/revista/pdf/artigos/878.pdf

13. Assunção TS, Ursine PGS. Estudo de fatores associados à adesão ao tratamento não farmacológico em portadores de diabetes mellitus assistidos pelo Programa Saúde da Família, Ventosa, Belo Horizonte. Ciênc Saúde Colet [serial on the internet]. 2008 [cited 2017 Dec 11];13(Suppl 2):218997. Available from: https://scielosp.org/pdf/csc/2008. v13suppl2/2189-2197/pt

14. Santos ECB, Teixeira CRS, Zanetti ML, Santos MA, Pereira MCA. Políticas públicas e direitos dos usuários do Sistema Único de Saúde com diabetes mellitus. Rev Bras Enferm [serial on the internet]. 2011 [cited 2017 Dec 11];64(5):952-7. Available from: http://www.scielo.br/pdf/reben/v64n5/ a23v64n5.pdf

15. Costa KS, Tavares NUL, Mengue SS, Pereira MA, Malta DC, Silva Júnior JB. Obtenção de medicamentos para hipertensão e diabetes no Programa Farmácia Popular do Brasil: resultados da Pesquisa Nacional de Saúde, 2013. Epidemiol Serv Saúde [serial on the internet]. 2016 [cited 2017 Dec 11];25(1):33-44. Available from: http://www.scielo.br/ scielo.php?pid=S2237-96222016000100033\&script $=$ sci abstract\&tlng=pt

16. Organização Pan-Americana da Saúde. Linhas de cuidado: hipertensão arterial e diabetes. Brasília (DF): OPAS; 2010.

17. Oliveira GKS, Oliveira ER. Assistência de enfermagem ao portador de diabetes mellitus: um enfoque na atenção primária em saúde. Veredas Favip: Revista Eletrônica de Ciências [serial on the internet]. 2010 [cited 2017 Dec 11];3(2):40-8. Available from: file:///D:/96-625-1-PB.pdf 
18. Cavalcante MLR, Viana LS, Filho RFS, Santos FCS, Brito MCC. Alimentação saudável e atividade física como condicionantes de síndromes metabólicas em adolescentes. Sanare (Sobral, Online) [serial on the internet]. 2016 [cited 2017 Dec 11];15(2):47-53. Available from: https://sanare. emnuvens.com.br/sanare/article/view/1037/583

19. Ben AJ, Neumann CR, Mengue SS. Teste de MoriskyGreen e Brief Medication Questionnaire para avaliar adesão a medicamentos. Rev Saúde Pública [serial on the internet]. 2012 [cited 2017 Dec 11];46(2):279-89. Available from: http://www.scielo.br/pdf/rsp/v46n2/3357.pdf

20. Araújo MF, Damasceno MMC, Gonçalves TC, Caetano JA. Aderência de diabéticos ao tratamento medicamentoso com hipoglicemiantes orais. Esc Anna Nery Rev Enferm [serial on the internet]. 2010 [cited 2017 Dec 11];14(2):361-7. Available from: http://www.scielo.br/pdf/ean/v14n2/20.pdf

21. Roos AC, Baptista DR, Miranda RC. Adesão ao tratamento de pacientes com diabetes mellitus tipo 2. Demetra [serial on the internet]. 2015 [cited 2017 Dec 11];10(2):329-46. Available from: http://www.e-publicacoes.uerj.br/index. php/demetra/article/view/13990/13277\#.WjAC3FWnGUk

22. Magnabosco P, Teroka EC, Oliveira EM, Felipe EA, Freitas $D$, Alves LMM. Análise comparativa da não adesão ao tratamento medicamentoso da hipertensão arterial sistêmica em população urbana e rural. Rev Latinoam Enferm [serial on the internet]. 2015 [cited 2017 Dec 11];23(1):20-7. Available from: http://www.revistas.usp.br/rlae/article/ view/100026/98617

23. Gimenes HT, Zanetti ML, Haas VJ. Fatores relacionados a adesão do paciente diabético a terapêutica medicamentosa. Rev Latinoam Enferm [serial on the internet]. 2009 [cited 2017 Dec 11];17(1). Available from: http://www.scielo.br/ pdf/rlae/v17n1/pt 08.pdf

24. Mendes LVP, Luiza VL, Campos MR. Uso racional de medicamentos entre indivíduos com diabetes mellitus e hipertensão arterial no município do Rio de Janeiro, Brasil. Ciênc Saúde Colet [serial on the internet]. 2014 [cited 2017 Dec 11];19(6):1673-84. Available from: http://www.scielo. $\underline{\mathrm{br} / \mathrm{pdf} / \mathrm{csc} / \mathrm{v} 19 \mathrm{n} 6 / 1413-8123-\mathrm{csc}-19-06-01673 . \mathrm{pdf}}$

25. Remondi FA, Cabrera MAS, Souza RKT. Não adesão ao tratamento medicamentoso contínuo: prevalência e determinantes em adultos de 40 anos e mais. Cad Saúde Pública [serial on the internet]. 2014 [cited 2017 Dec 11];30(1):126-36. Available from: http://www.scielo.br/ $\mathrm{pdf} / \mathrm{csp} / \mathrm{v} 30 \mathrm{n} 1 / 0102-311 \mathrm{X}$-csp-30-01-00126.pdf

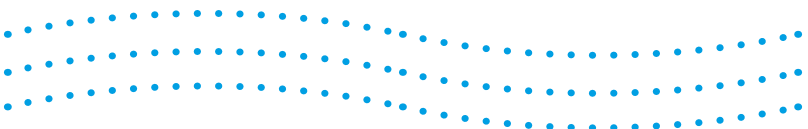

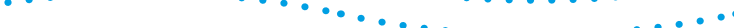

$\ldots \ldots \ldots \ldots \ldots \ldots \ldots \ldots$

$\ldots \ldots \ldots \ldots \ldots \ldots \ldots$

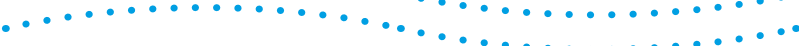

$\ldots \ldots \ldots \ldots$

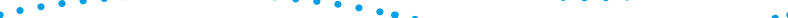

$\ldots \ldots \ldots \ldots \ldots \ldots \ldots \ldots \ldots \ldots$

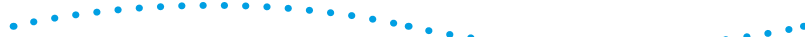
$\ldots \ldots \ldots \ldots \ldots \ldots \ldots \ldots$ $\ldots \ldots \ldots \ldots \ldots \ldots \ldots \ldots \ldots \ldots \ldots$ $\ldots \ldots \ldots \ldots \ldots \ldots$ $\ldots \ldots \ldots \ldots \ldots \ldots \ldots \ldots$ $\ldots \ldots \ldots \ldots \ldots \ldots \ldots \ldots \ldots \ldots$

Recebido em 22/06/2017 Aprovado em 27/11/2017

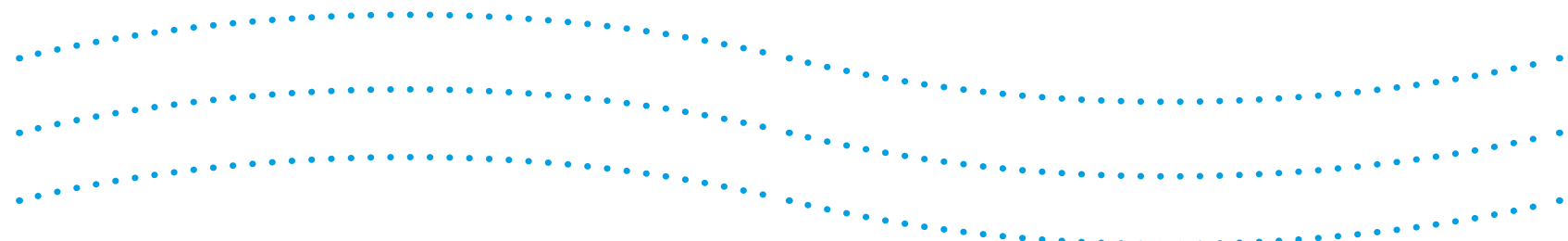

30 - SANARE, Sobral - V.16 n.02,p.22-30, Jul./Dez. - 2017 